\title{
Historical Development of (Modern) Meteoritics and Cosmochemistry
}

\section{HAP MCSWEEN}

University of Tennessee

Presenting Author: mcsween@utk.edu

Although there is a prehistory, involving the first recognition (Chladni, 1794) of meteorites as extraterrestrial objects and their initial chemical characterization (Howard, 1802), cosmochemistry is a relatively young discipline and thus my emphasis on "modern" milestones.

Among the bedrock advances in cosmochemistry (those that have shaped the discipline and have continued to develop over time), and their initial or perhaps most notable early proponents, are:

- Composition of the Sun and the solar system: cosmic abundances of the elements (Harkins, 1917; Goldschmidt, 1937), solar composition and carbonaceous chondrites as proxies (Suess and Urey, 1956), Genesis mission (returned solar wind samples in 2004)

- Compositions of planets: constraints from bulk density and precursor materials (Urey, 1952; Morgan and Anders, 1980; Wänke, 1981), lunar samples from Apollo missions (1970s), martian meteorites (McSween and Stolper, 1980; Bogard, 1983) and Mars rovers (1984+)

- Early solar system chronology: radiometric ages of Earth, Moon, and meteorites from ${ }^{207 / 206} \mathrm{~Pb}-\mathrm{Pb}$ (Patterson, 1956), ${ }^{87} \mathrm{Rb}-{ }^{87} \mathrm{Sr}$ (Compston, Wasserburg, 1960s), ${ }^{40 / 30} \mathrm{Ar}$ (Merrihue and Turner, 1966); ${ }^{147} \mathrm{Sm}^{-143} \mathrm{Nd}$ (Lugmair, DePaulo and Wasserburg, 1970s), short-lived ${ }^{129} \mathrm{I}$ (Reynolds, 1960), ${ }^{26} \mathrm{Al}$ (Lee, Wasserburg, 1977), ${ }^{182} \mathrm{Hf}$ (Halliday, 1995)

- Classification of chondrites: bringing order out of chaos, recognition of primary versus secondary properties (Prior, 1920; Keil and Fredrickson, 1965; Van Schmus and Wood, 1967)

- Volatility as a controlling factor in cosmochemical behavoir: volatile element depletions, condensation calculations and refractory inclusions (Larimer and Anders, 1967; Grossman, 1972)

- Nucleosynthesis and presolar grains: identification of presolar diamond and constraints on stellar nucleosynthesis (Burbage, Burbage, Fowler, Hoyle, 1964; Lewis, Anders, 1987; Amari, Anders, 1990)

- Classification of differentiated meteorites: irons (Wasson, 1967; Buchwald, 1975), magmatic and primitive achondrites as melts/cumulates and residues (Goodrich and Delaney, 2000)

- Organic cosmochemistry: first recognition in Orgueil (Cloez, 1864); first modern extractions from chondrites (Mueller, 1953; Vdovykin, 1964)

- Nucleosynthetic isotope anomalies and recognition of solar system reservoirs: (Clayton, 1973; Niederer, 